\title{
Originales
}

\section{Lavado broncoalveolar para el diagnóstico de neumonía en enfermos en ventilación mecánica}

\author{
ÁNGEL ESTELLA, M. IGNACIO MONGE, LUIS PÉREZ FONTAIÑA, ANDRÉS SAINZ DE BARANDA, M. \\ JESÚS GALÁN Y ELVIRA MORENO
}

Unidad de Cuidados Intensivos. Hospital del SAS de Jerez. Jerez. Cádiz. España.

Objetivo. Evaluar la utilidad diagnóstica del lavado broncoalveolar (LBA) en enfermos en ventilación mecánica con sospecha de neumonía y describir los resultados clínicos de los diferentes tipos de neumonía en enfermos críticos.

Diseño. Estudio descriptivo.

Ámbito. Unidad de cuidados intensivos (UCI) médico-quirúrgica de 17 camas.

Pacientes. Enfermos ingresados en $\mathrm{UCl}$ desde noviembre de $\mathbf{2 0 0 3}$ a marzo de 2006 con ventilación mecánica y sospecha clínica de neumonía a quienes se realizó LBA.

Intervenciones. Se realizó LBA a través de tubo orotraqueal con $150 \mathrm{ml}$ de suero salino fisiológico, repartidos en tres alícuotas de $50 \mathrm{ml}$; la muestra se procesó para estudio microbiológico.

Principales variables de interés. Edad, APACHE II al ingreso, días en ventilación mecánica, estancia en $\mathrm{UCl}$, mortalidad y agentes etiológicos aislados en el LBA.

Resultados. Se incluyó a 96 pacientes a quienes se realizó LBA; se distinguieron 4 grupos: neumonía adquirida en la comunidad (NAC), 12 casos; neumonía asociada a ventilación mecánica (NAVM) precoz, 26 casos. NAVM tardía, 43 casos, y neumonía en enfermos inmunodeficientes, 15 casos. El LBA fue positivo (> $10.000 \mathrm{ufc} / \mathrm{ml}$ ) en 40 $(41,7 \%)$ pacientes ( 2 con NAC, 16 con NAVM precoz, 17 con NAVM tardía, 5 con neumonía e inmunodeficiencia). La mortalidad fue del 33,3, el 26,9, el 25,6 y el $73,3 \%$ en NAC, NAVM precoz, NAVM tardía e inmunodeficientes, respectivamente.

Conclusiones. La escasa sensibilidad del LBA en la NAC corrobora la actitud de limitar su indicación sólo a casos seleccionados. En el grupo de enfermos inmunodeficientes la mortalidad fue

Correspondencia: Dr. A. Estella García.

Algarve, 27, 1. ${ }^{\circ}$ B. 11403 Jerez. Cádiz. España.

Manuscrito aceptado el 24-3-2008. muy elevada. EI LBA, según nuestra limitada experiencia, tiene su mayor utilidad en el diagnóstico de NAVM.

PALABRAS CLAVE: Neumonía. Lavado broncoalveolar. Ventilación mecánica.

\section{BRONCHOALVEOLAR LAVAGE FOR DIAGNOSING PNEUMONIA IN MECHANICALLY VENTILATED PATIENTS}

Objective. To evaluate the diagnostic role of bronchoalveolar lavage (BAL) in mechanically ventilated patients with suspected pneumonia and to describe the clinical outcome in the different kinds of pneumonia in critically ill patients.

Design. Descriptive study.

Setting. A 17-bed medical and surgical intensive care unit.

Patients. Mechanically ventilated patients admitted to the ICU from November 2003 to March 2006 with suspected pneumonia who underwent bronchoscopy with BAL.

Interventions. BAL was performed by fiberoptic bronchoscopy with three aliquots of $50 \mathrm{ml}$ sterile normal saline. Recovered BAL fluid was processed for microbiologic analysis.

Main variables of interest. Age, APACHE II score within the first $\mathbf{2 4}$ hours of admission, time on mechanical ventilation, ICU length of stay, mortality, and isolated bacteria were analyzed.

Results. A total of 96 cases of suspected pneumonia with BAL were recruited, including 4 groups: community associated pneumonia (CAP), 12 cases, early-onset ventilator-associated pneumonia (VAP), 26 cases, late-onset ventilator-associated pneumonia, 43 cases, and immunocompromised patients, 15 cases. BAL was positive (> $10000 \mathrm{ufc} / \mathrm{ml})$ in $40(41.7 \%)$ patients $(2,16,17$ and 5 patients with CAP, early-onset VAP, late-onset 
VAP and immunocompromised, respectively). Mortality was $33.3 \%, 26.9 \%, 25.6 \%$ and $73.3 \%$ in CAP, early-onset VAP, late-onset VAP and immunocompromised patients respectively.

Conclusions. The low incidence of positive BAL in the CAP group supports using BAL only for particularly severe, selected cases. Mortality was very high in the immunocompromised patients. In the light of our personal experience, BAL is most useful in the diagnosis of pneumonia in the group of patients with VAP.

KEY WORDS: Pneumonia. Bronchoalveolar lavage. Mechanical ventilation.

\section{INTRODUCCIÓN}

La neumonía en pacientes críticos es una enfermedad de gran importancia en las unidades de cuidados intensivos (UCI), no sólo por su elevada mortali$\mathrm{dad}^{1,2}$, sino también por su gran incidencia. La neumonía asociada a ventilación mecánica (NAVM) es la infección nosocomial más frecuente en las $\mathrm{UCI}^{3-5}$. Conlleva mayor tiempo en ventilación mecánica, mayor estancia en UCI y en el hospital ${ }^{6,7}$ y gran consumo de antibióticos ${ }^{8}$.

A pesar de los numerosos estudios realizados, el método para el diagnóstico de neumonía en pacientes en ventilación mecánica sigue siendo objeto de debate $\mathrm{e}^{9,10}$, y se distinguen dos estrategias diagnósticas: la «no invasiva», basada en criterios clínicos y cultivos de vías respiratorias altas ${ }^{10,11}$, y la estrategia «invasiva», basada en el uso de técnicas broncoscópicas para el diagnóstico microbiológico mediante cultivos cuantitativos de muestras obtenidas del tracto respiratorio inferior ${ }^{11-13}$. Los cultivos microbiológicos cualitativos tienen una alta sensibilidad y una moderada especificidad, ésta aumenta con el uso de cultivos cuantitativos. Según las recomendaciones elaboradas por las sociedades científicas SEPAR, SEIMC y SEMICYUC, el aspirado traqueal cualitativo no se debe utilizar de forma sistemática para el diagnóstico de NAVM; es más recomendable el uso de técnicas de cultivo cuantitativas, obtenidas mediante procedimientos invasivos (técnicas broncoscópicas) o no invasivos (broncoaspirado), en función de la experiencia del médico y de los medios disponibles ${ }^{14}$.

Hemos realizado un estudio en pacientes ventilados mecánicamente en UCI, en los que se sospechó neumonía, con el objetivo de evaluar la rentabilidad diagnóstica del lavado broncoalveolar (LBA) en los diferentes tipos de neumonías (adquirida en la comunidad, NAVM precoz y tardía y neumonía en pacientes inmunodeficientes), así como describir los resultados clínicos.

\section{PACIENTES Y MÉTODOS}

Se trata de un estudio descriptivo realizado en una UCI médico-quirúrgica de 17 camas, en el que se incluyó a pacientes consecutivos ingresados en UCI desde noviembre de 2003 a marzo de 2006, que recibieron ventilación mecánica, en los que hubo sospecha clínica de neumonía, basada en la imagen de infiltrado en la radiografía de tórax y al menos dos de los siguientes criterios: $a$ ) fiebre de más de $38,3{ }^{\circ} \mathrm{C}$; b) secreciones traqueobronquiales purulentas, y $c$ ) leucocitosis $>12.000 / \mu$ l o leucopenia $<4.000 / \mu 1$.

Distinguimos cuatro tipos de neumonías: neumonía adquirida en la comunidad (NAC), NAVM pre$\mathrm{coz}^{15}$, definida como aquella que se presenta en los primeros 4 días en ventilación mecánica, NAVM tardía ${ }^{15}$, aquella que se presenta a partir del quinto día en ventilación mecánica, y neumonía en enfermos inmunodeficientes.

Los criterios de exclusión fueron: tubo orotraqueal $<8 \mathrm{~mm}$, neumotórax, $\mathrm{SatO}_{2}<90 \%$ con $\mathrm{FIO}_{2}$ de 1 , acidosis severa: $\mathrm{pH}<7,20$.

Se analizaron las siguientes características clínicas: edad, APACHE II al ingreso, días en ventilación mecánica, estancia en UCI, mortalidad, antibioterapia previa y agentes etiológicos aislados en el LBA.

Se realizó LBA con $150 \mathrm{ml}$ de suero salino fisiológico, repartidos en tres alícuotas de $50 \mathrm{ml}$, e instilados en la localización indicada por la radiografía de tórax o a nivel del lóbulo medio derecho en el caso de infiltrado bilateral. No se administraron anestésicos locales y se evitó la aspiración a través del canal del broncoscopio hasta enclavarlo en la localización a estudiar. Al realizar el LBA, los primeros $20 \mathrm{ml}$ recuperados fueron desechados y una muestra del líquido restante fue procesado para estudio microbiológico: tinción directa de la muestra (tinción de Gram), tinción para micobacterias y hongos, cultivo bacteriano en los medios habituales (agar sangre, agar chocolate, MacConkey, agar Brucella y BHI) y en medios específicos para Mycobacteria (medio líquido: Bactec MGIT-960BD), hongos (agar Sabouraud) y Legionella (BCYE). En enfermos inmunodeficientes se realizó tinción específica para Pneumocystis jiroveci. También se aplicaron técnicas de inmunofluorescencia para Legionella. No se realizó estudio citológico.

El modo de ventilación fue presión-control, con $\mathrm{FiO}_{2}$ de 1 . El LBA se consideró positivo con crecimiento de más de $10.000 \mathrm{ufc} / \mathrm{ml}$.

Los datos recogidos fueron analizados mediante el programa estadístico SPSS versión 15.0 para Windows. Se consideró significativo un valor de $\mathrm{p}<$ 0,05 . Las variables cuantitativas se expresan en media y desviación estándar, y las variables cualitativas, en porcentaje. Para comparar variables cuantitativas entre dos grupos se aplicó la prueba de la t de Student en los casos en que podemos asumir normalidad, y la prueba no paramétrica de Mann-Whitney si no se podía asumir normalidad en la distribución de la muestra; se aplicó la prueba de la $\chi^{2}$ para comparar variables cualitativas.

\section{RESULTADOS}

Durante el período de estudio 683 pacientes precisaron de ventilación mecánica. La incidencia de NAVM diagnosticada con criterios clínicos fue de un 
TABLA 1. Características de los pacientes con sospecha de neumonía incluidos en el estudio

\begin{tabular}{|l|c|c|c|}
\hline & NAC $(\mathrm{n}=12)$ & NAVM precoz $(\mathrm{n}=26)$ & $\begin{array}{c}\text { NAVM tardía }(\mathrm{n}=43) \\
\text { Pacientes inmunodeficientes } \\
(\mathrm{n}=15)\end{array}$ \\
\hline Edad, años & $55,7 \pm 19,8$ & $52,1 \pm 14,2$ & $63,6 \pm 14,3$ \\
APACHE II & $19,6 \pm 8,5$ & $18,4 \pm 7$ & $20,5 \pm 8,4$ \\
Empleo de antibióticos previos & $10(83,3 \%)$ & $17(65,4 \%)$ & $22,4 \pm 3,7$ \\
LBA positivo & $2(16,7 \%)^{\mathrm{a}}$ & $16(61,5 \%)$ & $35(81,4 \%)$ \\
Duración de la VM, días & $12,1 \pm 8,3^{\mathrm{b}}$ & $6,7 \pm 5,2$ & $17(39,5 \%)$ \\
Estancia en UCI, días & $14,2 \pm 8,3$ & $10,2 \pm 7$ & $16,8 \pm 10,6^{\mathrm{b}}$ \\
Mortalidad & $4(33,3 \%)$ & $7(26,9 \%)$ & $23,2 \pm 13,2^{\mathrm{b}}$ \\
\hline
\end{tabular}

LBA: lavado broncoalveolar; NAC: neumonía adquirida en la comunidad; NAVM: neumonía asociada a ventilación mecánica; UCI: unidad de cuidados intensivos. ${ }^{a}$ En el LBA se identificó, mediante inmunofluorescencia directa, Legionella pneumophila en 2 casos.

${ }^{b} \mathrm{p}<0,05$ con respecto a NAVM precoz.

c $\mathrm{p}<0,05$ con respecto a NAC y NAVM.

Los datos se presentan como media \pm desviación estándar o n (\%).

10,1\%. Se realizó LBA a todos los pacientes con sospecha clínica de NAVM o inmunodeficientes.

Durante el período de estudio ingresaron en UCI 51 pacientes con NAC; en 10 casos que presentaron mala respuesta al tratamiento antibiótico inicial se realizó LBA y en 2 casos que presentaron una evolución de curso fulminante al ingreso en UCI se realizó LBA inmediatamente antes de iniciar tratamiento antibiótico empírico.

Se excluyó a 5 pacientes por presentar tubo orotraqueal $<8 \mathrm{~mm}$ de diámetro (3 casos), o hipoxemia a pesar de $\mathrm{FiO}_{2}$ de 1 (2 casos).

Este estudio incluye a 96 pacientes consecutivos en ventilación mecánica en los que hubo sospecha clínica de neumonía: 12 casos con NAC, 26 casos con NAVM precoz, 43 casos con NAVM tardía y 15 casos de neumonía en enfermos inmunodeficientes. En la tabla 1 se exponen las características de los pacientes según el tipo de neumonía.

TABLA 2. Microorganismos aislados en el lavado broncoalveolar (> $10.000 \mathrm{ufc} / \mathrm{ml}$ )

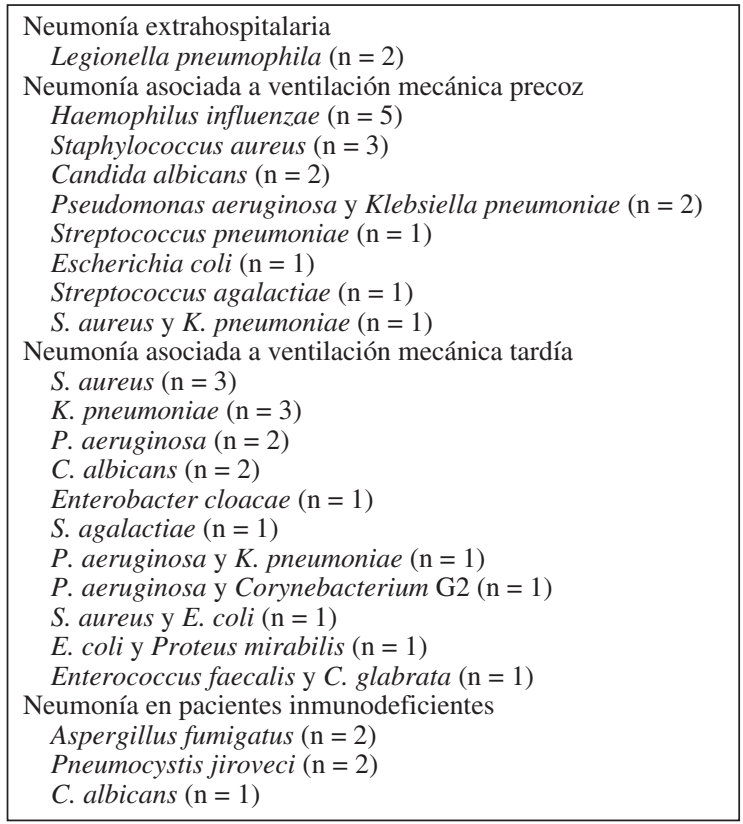

Antes de la realización del LBA, $75(78,1 \%)$ pacientes recibían antibióticos. El LBA fue positivo (> $10.000 \mathrm{ufc} / \mathrm{ml})$ en $40(41,7 \%)$ pacientes. La rentabilidad diagnóstica del LBA osciló entre un 16,7\% en el grupo de NAC y un $61,5 \%$ en el grupo de enfermos con NAVM precoz, que corresponde al grupo con menor porcentaje de antibioterapia previa $(65,4 \%)$ (tabla 1).

El diagnóstico etiológico fue diferente en los distintos tipos de neumonía (tabla 2). Haemophilus influenzae fue el microorganismo más frecuentemente aislado en el grupo de NAVM precoz, los microorganismos gramnegativos fueron los más frecuentemente aislados en el LBA del grupo de NAVM tardías, y las infecciones por microorganismos oportunistas fueron las más frecuentes en el grupo de inmunodeficientes.

Se compararon los resultados microbiológicos positivos entre los enfermos según hubieran recibido antibioterapia previa o no y se observó que aqullos sin antibioterapia presentaban una proporción significativamente mayor de resultados microbiológicos positivos (tabla 3 ).

\section{DISCUSIÓN}

El diagnóstico etiológico de las NAC que requieren ingreso hospitalario se obtiene sólo en un 40-60\% de los pacientes ${ }^{16}$; un $50 \%$ de las que ingresan en UCI están causadas por Streptoccocus pneumoniae y Legionella spp. ${ }^{17,18}$. Las técnicas diagnósticas invasivas no son una indicación habitual, sólo están indicadas en las NAC de curso fulminante o que no responden al tratamiento antimicrobiano empírico inicial ${ }^{19}$. En nuestro estudio, el bajo porcentaje de LBA positivo en el grupo de NAC podría explicarse porque la antibioterapia previa estaba dirigida al tratamiento de una infección pulmonar, a diferencia de los grupos de NAVM e inmunodeficientes en los que se había prescrito antibioterapia por otros motivos. Por otra parte, la introducción reciente de antibióticos disminuye la rentabilidad diagnóstica del LBA, y en el grupo de enfermos con NAC se había introducido en las últimas $48 \mathrm{~h}$ previas a la toma de muestras.

El subgrupo de enfermos inmunodeficientes constituye una muestra muy heterogénea respecto al resto de 
TABLA 3. Tabla de contingencia comparativa entre los resultados del lavado broncoalveolar (LBA) según el empleo de antibioterapia previa

\begin{tabular}{|l|c|c|c|}
\hline & $\begin{array}{c}\text { Sin antibioterapia } \\
\text { previa }\end{array}$ & $\begin{array}{c}\text { Con antibioterapia } \\
\text { previa }\end{array}$ & Total \\
\hline LBA positivo & 14 & 26 & 40 \\
LBA negativo & 7 & 49 & 56 \\
Total & 21 & 75 & 96 \\
\hline
\end{tabular}

Prueba de la $\chi^{2}, p=0,009$.

los pacientes analizados, debido a su mayor susceptibilidad a presentar infecciones a causa de su enfermedad de base y/o tratamiento. A pesar del tratamiento antibiótico empírico de amplio espectro, la mortalidad en estos pacientes es muy elevada ${ }^{20}$, en nuestra serie tres cuartas partes de este grupo fallecieron.

La mayor rentabilidad diagnóstica del LBA en nuestra serie se ha obtenido en el subgrupo de NAVM, especialmente en la precoz, subgrupo que presentaba una menor proporción de pacientes con antibioterapia previa, la cual disminuye la sensibilidad del $\mathrm{LBA}^{21}$. Al comparar los resultados del LBA en los pacientes con o sin antibioterapia previa, destacó de forma estadísticamente significativa la mayor rentabilidad obtenida cuando el LBA se realizaba en aquellos sin tratamiento previo con antibióticos.

La estancia media en UCI fue mayor en el grupo con NAVM tardía. El riesgo estimado de NAVM es del 3\%/día en los primeros 5 días, el 2\%/día en 5-10 días y el 1\%/día en los días siguientes ${ }^{2}$, ello explicaría que a más días en ventilación mecánica mayor riesgo de presentar NAVM y, a su vez, debido a esta infección nosocomial, mayor estancia hospitalaria.

La mortalidad observada en nuestra serie en los distintos tipos de neumonía coincide con la descrita en otros estudios ${ }^{22-24}$. Sin embargo, hay una gran heterogeneidad en cuanto a mortalidad en la literatura ${ }^{15}$, probablemente explicada no sólo por las diferentes poblaciones y patógenos aislados en cada estudio, sino por la falta de consenso en los métodos a utilizar para el diagnóstico de neumonía.

No hay una prueba definitiva para el diagnóstico de neumonía; en pacientes en ventilación mecánica se diagnostica por la asociación de criterios clínicos, radiológicos y microbiológicos. Los criterios clínicos por sí solos tienen escasas sensibilidad y especifici$\mathrm{dad}^{25}$, la radiografía de tórax puede llegar a ser muy inespecífica, no sólo por la baja calidad obtenida con aparatos portátiles, sino por la variedad de causas que se expresan radiológicamente como infiltrado alveolar (atelectasias, disnea, edema agudo de pulmón, hemorragia pulmonar...) en los pacientes críticos en ventilación mecánica.

A pesar de las recomendaciones de la Sociedad Española de Medicina Intensiva en el diagnóstico de $\mathrm{NAVM}^{26}$, llama la atención la poca utilización de métodos diagnósticos invasivos con cultivos cuantitativos en las UCI españolas. Recientemente se ha publicado un estudio respecto a la prevención y el diagnóstico de NAVM en 32 UCI de Andalucía en el que destaca el escaso porcentaje de empleo de técnicas broncoscópicas para el diagnóstico de NAVM, tan sólo 4 (14,3\%) de los hospitales encuestados utilizaban técnicas broncoscópicas ${ }^{27}$. En el estudio nacional de vigilancia de infección nosocomial en servicios de medicina intensiva españoles (ENVIN) sólo en una tercera parte de las NAVM se utilizaron métodos invasivos para su diagnóstico, en el informe del año 2006, y tan sólo un 9,21\% utilizó el lavado broncoalveolar como método diagnóstico ${ }^{28}$.

La principal limitación del aspirado traqueal es que no diferencia entre colonización e infección, el aspirado traqueal cualitativo no es recomendado para el diagnóstico microbiológico de $\mathrm{NAVM}^{2,14}$, a pesar de ello fue el método más utilizado en las UCI de Andalucía $^{27}$, donde la incidencia de NAVM es alta ${ }^{29}$. Basándonos en este método diagnóstico cabe la posibilidad de que estemos sobrediagnosticando neumonías que realmente no lo son, tratándose de colonizaciones traqueales o traqueobronquitis.

No hay consenso respecto a la utilidad de la broncoscopia en los enfermos críticos. Leroy et $\mathrm{al}^{30}$ destacan que no se puede considerar las técnicas broncoscópicas como un procedimiento sistemático en UCI; sin embargo, disponer del broncoscopio en UCI, tanto para procedimientos diagnósticos como terapéuticos de urgencia, está avalado por distintos autores ${ }^{31}$, es una técnica sencilla, se realiza a pie de cama y presenta pocas complicaciones. En el caso de no disponer de fibrobroncoscopia o personal experimentado para realizar el procedimiento, en los últimos años se han desarrollado técnicas invasivas no broncoscópicas para la obtención de muestras de vías respiratorias bajas cuyos cultivos cuantitativos presentan sensibilidad y especificidad similares a las obtenidas con la broncoscopia. Fagon et $\mathrm{al}^{10}$, en un estudio multicéntrico aleatorizado que comparó las dos estrategias diagnósticas, invasiva y no invasiva, concluyeron que la estrategia broncoscópica conlleva una reducción de la mortalidad a los 14 días. La ATS incluye el diagnóstico broncoscópico con un nivel de evidencia I en sus recomendaciones ${ }^{2}$. En un estudio aleatorizado en 740 pacientes críticos con sospecha de NAVM, se observó que los resultados clínicos y la administración de antibióticos eran iguales, independientemente de que se utilizara la estrategia invasiva o $\mathrm{no}^{32}$. Una importante limitación de ese estudio es que se excluyó a enfermos inmunodeficientes, con enfermedades crónicas, con tratamiento con carbapenem o quinolonas y con colonización de microorganismos resistentes a antibioterapia; todas estas exclusiones, un $40 \%$ de los pacientes del estudio, representan el perfil de la mayoría de los pacientes ingresados en la UCI con sospecha clínica de NAVM.

El empleo de técnicas broncoscópicas no sólo es una recomendación de las guías de diferentes sociedades para el diagnóstico de neumonías ${ }^{2,26}$, sino que además es de gran utilidad, cuando el lavado broncoalveolar no ha sido positivo, para redirigir la búsqueda de otros posibles focos infecciosos de origen extrapulmonar $^{11} \mathrm{u}$ otras causas no infecciosas ${ }^{33}$ en el paciente crítico. 
La principal limitación de este estudio es que incluye pocos pacientes y que la mayoría de ellos presentaban antibioterapia previa. Basados en nuestra limitada experiencia, concluimos que la escasa sensibilidad del LBA en la NAC corrobora que su indicación debe estar limitada sólo a casos seleccionados; el LBA tiene su mayor utilidad en el diagnóstico de NAVM. Conocer la etiología más frecuente de NAVM en cada UCI proporciona una herramienta muy valiosa para el manejo antibiótico empírico inicial y posterior ajuste cuando se obtengan los resultados microbiológicos.

\section{BIBLIOGRAFÍA}

1. Moine P, Vercken JB, Chevret S, Chastang C, Gagdos P. Severe community-acquired pneumonia. Etiology, epidemiology, and prognosis factors. Chest. 1995; 107:1182-3.

2. American Thoracic Society Documents. Guidelines for the Management of Adults with Hospital-acquired, Ventilator-associated, and Healthcare-associated Pneumonia. Am J Respir Crit Care Med. 2005;171:388-416.

3. Richards MJ, Edwards JR, Culver DH, Gaynes RP. Nosocomial infections in combined medical-surgical intensive care units in the United States. Infect Control Hosp Epidemiol. 2000;21:510-5.

4. Vincent JL, Bihari DJ, Suter PM, Bruining HA, White J, Nicolas-Chanion $\mathrm{MH}$, et al. The prevalence of nosocomial infection in Intensive Care Units in Europe. Results of the European Prevalence of Infection in Intensive Care (EPIC) study. JAMA. 1995;274:639-44.

5. Davis KA. Ventilator-associated pneumonia: a review. J Intensive Care Med. 2006;21:211-26.

6. Olaechea PM, Ulibarrena MA, Álvarez-Lerma F, Insausti J, Palomar M, De la Cal MA. The ENVIN-UCI Study Group. Factors related to hospital stay among patients with nosocomial infection acquired in the Intensive Care Unit. Infect Control Hosp Epidemiol. 2003;24:207-13.

7. Rello J, Ollendorf DA, Oster G, Vera-Llonch M, Bellm L, Redman R, et al. Epidemiology and outcomes of ventilator-associated pneumonia in a large US Database. Chest. 2002;122:2115-21.

8. Richards MJ, Edwards JR, Culver DH, Gaynes RP. Nosocomial infections in medical intensive care units in the United States. National Nosocomial Infections Surveillance System. Crit Care Med. 1999;27:887-92.

9. Shorr AF, Sherner JH, Jackson WL, Kollef MH. Invasive approaches to the diagnosis of ventilator-associated pneumonia: a meta-analysis. Crit Care Med. 2005;33:46-53.

10. Fagon JY, Chastre J, Wolff M, Gervais C, Parer-Aubas S, Stephan F, et al. Invasive and noninvasive strategies for management of suspected ventilator-associated pneumonia. A randomized trial. Ann Intern Med. 2000;132:621-30.

11. Fagon JY. Diagnosis and treatment of ventilator-associated pneumonia: fiberoptic bronchoscopy with bronchoalveolar lavage is essential. Semin Respir Crit Care Med. 2006;27:34-44.

12. Timsit JF, Cheval C, Gachot B, Bruneel F, Wolff M, Carlet $\mathrm{J}$, et al. Usefulness of a strategy based on bronchoscopy with direct examination of bronchoalveolar lavage fluid in the initial antibiotic therapy of suspected ventilator-associated pneumonia. Intensive Care Med. 2001;27:640-7.

13. Vallés J, Rello J, Fernández R, Blanch L, Baigorri F, Mestre $\mathrm{J}$, et al. Role of bronchoalveolar lavage in mechanically ventilated patients with suspected pneumonia. Eur J Clin Microbiol Infect Dis. 1994;13:549-58.

14. Álvarez F, Torres A, Rodríguez de Castro F. Recomendaciones para el diagnóstico de la neumonía asociada a ventilación mecánica. Med intensiva. 2001;7:271-82.

15. Chastre J, Fagon JY. Ventilator-associated pneumonia. Am J Respir Crit Care Med. 2002;165:867-903.

16. Woodhead M. Community-acquired pneumonia in Europe: causative pathogens and resistance patterns. Eur Respir J. 2002;20:20-7.

17. Rello J, Quintana A, Ausina V, Net A, Prats G. A three-year study of severe community acquired pneumonia with emphasis in outcome. Chest. 1993;103:232-5.

18. Leroy O, Santré C, Beuscart C, Georges H, Guery B, Jacquier JM, et al. A five-year study of severe community acquired pneumonia with emphasis on prognosis on patients admitted to an intensive care unit. Intensive Care Med. 1995; 21:24-31

19. Normativas para el diagnóstico y el tratamiento de la neumonía adquirida en la comunidad. Sociedad Española de Neumología y Cirugía Torácica (SEPAR). Arch Bronconeumol. 2005;41:272-89.

20. Gruson D, Hilbert G, Valentino R, Vargas F, Chene G, Bebear C, et al. Utility of fiberoptic bronchoscopy in neutropenic patients admitted to the intensive care unit with pulmonary infiltrates. Crit Care Med. 2000;28:2224-30.

21. Bouza E, Torres MV, Burillo A. Aportación del laboratorio de microbiología al diagnóstico de la neumonía asociada a ventilación mecánica. Enferm Infecc Microbiol Clin. 2005;23:2-9.

22. Gowardman J, Trent L. Severe community acquired pneumonia: a one-year analysis in a tertiary referral intensive care unit. NZ Med J. 2000;113:161-4.

23. Wilson PA, Ferguson J. Severe community-acquired pneumonia: an Australian perspective. Intern Med J. 2005;35: 699-705.

24. Luna CM, Vujacich P, Niederman MS, Vay C, Gherardi C, Matera J, et al. Impact of BAL data on the therapy and outcome of ventilator-associated pneumonia. Chest. 1997;111:676-85.

25. Fagon JY, Chastre J, Hance AJ, Domart Y, Trouillet JL, Gibert C. Evaluation of clinical judgment in the identification and treatment of nosocomial pneumonia in ventilated patients. Chest. 1993; 103:547-53.

26. Álvarez Lerma F, Álvarez-Sánchez B, Barcenilla F. Protocolo diagnóstico y terapéutico de la neumonía asociada a ventilación mecánica. Guía de práctica clínica en medicina intensiva. Sociedad Española de Medicina Intensiva y Unidades Coronarias. Barcelona: Meditex; 1996. p. 1-8.

27. Sierra R, Benitez E, Leon C, Rello J. Prevention and diagnosis of ventilator-associated pneumonia: a survey on current practices in Southern Spanish ICUs. Chest. 2005;128:1667-73.

28. Palomar M, Álvarez-Lerma F, De la Cal MA, Insausti J, Olaechea P, y Grupo de Estudio de Vigilancia de Infección Nosocomial en UCI (ENVIN-UCI). ICAAC, Toronto, septiembre de 2000.

29. Sierra Camerino R, Ortiz C. Prevalence of infections and systemic inflamatory response syndrome in southern spanish ICUs. Intensive Care Med. 2001;27:791-2.

30. Leroy O, Georges H, Boussekey N. Diagnosis and therapy of ventilator associated pneumonia. Int J Intensive Care. 2005;12:3.

31. British Thoracic Society. Guidelines on diagnostic flexible bronchoscopy. Thorax. 2001;56 Suppl 1:11-21.

32. The Canadian Critical Care Trial Group. A randomized trial of diagnostic techniques for ventilator associated pneumonia. $\mathrm{N}$ Engl J Med. 2006;355:2619-30.

33. Menendez R, Torres A. Treatment failure in community-acquired pneumonia. Chest. 2007;132:1348-55. 